\title{
Rechtliche Anforderungen an den Umgang mit Behandlungsdokumentationen bei Praxisaufgabe und Praxisverkauf
}

Die Wahrung der ärztlichen Schweigepflicht ist eine der substanziellen Pflichten im ärztlichen Berufsrecht, da sie das Vertrauensverhältnis zwischen Patient und Arzt nachhaltig prägt. Um es nicht zu gefährden, ist der korrekte Umgang mit der Behandlungsdokumentation sicherzustellen. Dies stellt den Behandelnden vor besondere Herausforderungen, wenn die Aufgabe oder der Verkauf seiner Praxis beabsichtigt wird. Es muss sichergestellt werden, dass die Patientenakte unter Wahrung des informationellen Selbstbestimmungsrechts des Patienten ordnungsgemäß an den Rechtsnachfolger übergeben wird oder Maßnahmen zur sachgerechten Aufbewahrung der Behandlungsdokumentationen ergriffen werden.

\section{Allgemeine Pflichten bei der Behandlungsdoku- mentation}

Die Pflichten zur Behandlungsdokumentation und zur Aufbewahrung der Patientenakte sind gesetzlich nicht einheitlich geregelt. Es stellt sich als Zusammenspiel verschiedener Normen dar. Während die Musterberufsordnung (MBO-Ä) die Pflicht zur Behandlungsdokumentation in $\S 10$ Abs. 1 noch weit fasst („Ärztinnen und Ärzte haben über die in Ausübung ihres Berufes gemachten Feststellungen und getroffenen Maßnahmen die erforderlichen Aufzeichnungen zu machen.") und der Umfang der Dokumentationspflicht durch den unbestimmten Rechtsbegriff der Erforderlichkeit für den Arzt nicht offenkundig ist, ist durch das Gesetz zur Verbesserung der Rechte von Patientinnen und Patienten vom 20.2.2013 (BGBI. I S. 277) in §630f BGB die Dokumentationspflicht und ihr Umfang für den Arzt transparent kodifiziert worden. $\S 630 f$ Abs. 1 S. 1 BGB verpflichtet den Behandelnden, „zum Zweck der Dokumentation in unmittelbarem zeitlichen Zusammenhang mit der Behandlung eine Patientenakte in Papierform oder elektronisch zu führen“. §630f Abs. 2 BGB konkretisiert den Umfang der Dokumentationspflicht dergestalt, dass „in der Patientenakte sämtliche aus fachlicher
Sicht für die derzeitige und künftige Behandlung wesentlichen Maßnahmen und deren Ergebnisse aufzuzeichnen [sind], insbesondere die Anamnese, Diagnosen, Untersuchungen, Untersuchungsergebnisse, Befunde, Therapien und ihre Wirkungen, Eingriffe und ihre Wirkungen, Einwilligungen und Aufklärungen “. Nachträgliche Korrekturen, Änderungen oder Ergänzungen müssen als solche gekennzeichnet sein. Für den Behandelnden ist nunmehr aus dem Gesetzestext eindeutig der Umfang seiner Dokumentationspflicht ersichtlich.

In der Radiologie werden an den Umfang der Dokumentationspflicht speziellere, zusätzliche Anforderungen geknüpft. Bei der Anwendung von Röntgenstrahlung am Menschen müssen die Behandlungsdokumentationen gemäß $\S 28$ Abs. 1 S. 2 RöV die Ergebnisse der rechtfertigenden Indikation, den Zeitpunkt und die Art der Anwendung, die untersuchte Körperregion, die Angaben zur rechtfertigenden Indikation, bei einer Untersuchung zusätzlich den erhobenen Befund, die Strahlenexposition des Patienten, die zu deren Ermittlung erforderlichen Daten und Angaben und bei einer Behandlung zusätzlich der Bestrahlungsplan sowie das Bestrahlungsprotokoll enthalten. Die Verordnung über den Schutz vor Schäden durch ionisierende Strahlen (StrlSchV) beinhaltet in $\S 85$ Abs. 1 S. 2 ebenfalls eine Aufzählung, die den Umfang der Aufzeichnungspflichten bei der Anwendung radioaktiver oder ionisierender Strahlung am Menschen konkretisiert.

Die Pflicht zur Behandlungsdokumentation obliegt grundsätzlich dem Behandelnden persönlich. Behandelnder ist derjenige, der die medizinische Behandlung eines Patienten zusagt und den Behandlungsvertrag mit dem Patienten abschließt. Ist ein Arzt in einer Einzelpraxis tätig, so ist er der Behandelnde des Patienten und hat die gesetzliche Pflicht, die Patientenakte ordnungsgemäß zu führen und aufzubewahren.

Ist der Arzt als Gesellschafter oder Angestellter in einer Gesellschaft in der Rechts-

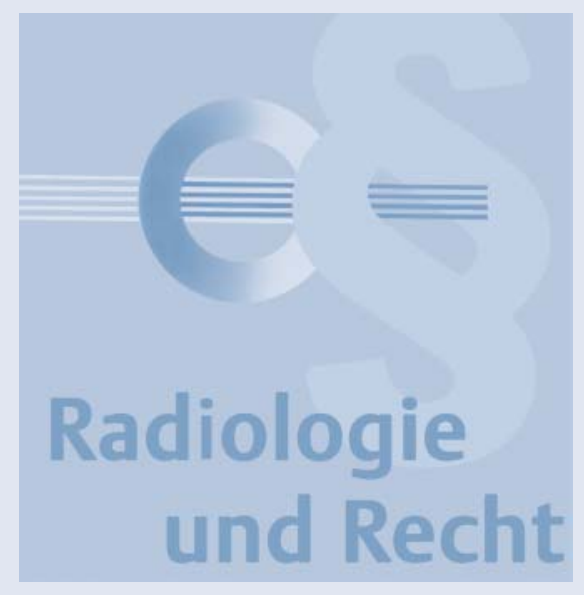

form einer Gesellschaft bürgerlichen Rechts (GbR), einer Partnerschaftsgesellschaft (PartG) oder einer Gesellschaft mit beschränkter Haftung (GmbH), die als Berufsausübungsgemeinschaft oder Medizinisches Versorgungszentrum kooperiert, tätig, so schließt der Patient mit der Gesellschaft den Behandlungsvertrag ab. Die Gesellschaft ist Vertragspartei des Patienten und als Behandelnde im Sinne des Gesetzes zu qualifizieren, wenngleich sie die Behandlung als solche durch einen Erfüllungsgehilfen, den behandelnden Arzt, leistet. Der Patient willigt bei Aufsuchen der Praxis zumindest konkludent ein, von allen Ärzten der Gesellschaft behandelt zu werden und gewährt damit jedem Einzelnen Zugriff auf seine Patientenakte. Eine Ausnahme besteht nur dann, wenn der Patient explizit nur die Behandlung von einem bestimmten Arzt einfordert. Die Gesellschaft verpflichtet sich vertraglich, die Pflichten aus dem Behandlungsvertrag zu erfüllen. Dies umfasst auch die Pflicht, durch den behandelnden Arzt die Behandlung nach Maßgabe der gesetzlichen Anforderungen zu dokumentieren. Als Behandelnde trifft die Gesellschaft als Vertragspartei des Patienten die Pflicht, die Patientenakte aufzubewahren. Sie ist Eigentümerin der Patientenakte.

Anders ist dies zu bewerten, wenn der Arzt in einer Praxisgemeinschaft (auch Organisationsgemeinschaft genannt), einem Zusammenschluss von Ärzten zur gemeinsamen Nutzung von Räumen, Einrichtungen oder Personal, tätig ist. In diesem Fall schließt der Patient nur mit dem ihn behandelnden Arzt den Behandlungsvertrag ab und nicht mit der Praxisgemeinschaft. Dies 
folgt aus der Tatsache, dass in einer Organisationsgemeinschaft jeder Arzt seine Praxis eigenständig führt und die Gesellschaft im Außenverhältnis nicht als einheitliche Praxis zu bewerten ist; es sei denn, sie tritt als solche nach außen hin auf (vgl. Ratzel in Spickhoff, Medizinrecht, 2. Auflage 2014, §705 BGB, Rn. 13). Im Rahmen einer Praxisgemeinschaft ist daher der behandelnde Arzt für die Erfüllung der Pflicht zur Behandlungsdokumentation und ordnungsgemäße Aufbewahrung der Patientenakte verantwortlich.

\section{Aufbewahrungsfristen}

Nach §630f Abs. 1 S. 1 BGB ist der Behandelnde verpflichtet, zum Zweck der Dokumentation in unmittelbarem zeitlichen $\mathrm{Zu}$ sammenhang mit der Behandlung eine Patientenakte in Papierform oder elektronisch zu führen. Die Patientenakte ist für die Dauer von zehn Jahren nach Abschluss der Behandlung aufzubewahren, soweit nicht in anderen Vorschriften andere Aufbewahrungsfristen bestehen (vgl. §630f Abs. 3 BGB, § 10 Abs. 3 MBO-Ä). Aufzeichnungen über Röntgenbehandlungen sind dreißig Jahre lang nach der letzten Behandlung aufzubewahren (vgl. §28 Abs. 3 S. 1 RöV). Darunter gefasst sind Niederschriften der Röntgentherapie. Gleiches gilt für Aufzeichnungen über die Behandlung bei der Anwendung radioaktiver Stoffe oder ionisierender Strahlung am Menschen (vgl. §85 Abs. 3 S. 1 StrlSchV). Röntgenbilder und die Aufzeichnungen über Röntgenuntersuchungen, mithin Unterlagen zur Röntgendiagnostik, sind zehn Jahre lang nach der letzten Untersuchung aufzubewahren (vgl. § 28 Abs. 3 S. 2 RöV). Die Aufzeichnungen müssen nach $\S 28$ Absatz 1 S. 2 Nr. 1 bis 7 die Ergebnisse der Befragung des Patienten nach $\S 23$ Abs. 2 S. 2 und Abs. 3 S. 1, den Zeitpunkt und die Art der Anwendung, die untersuchte Körperregion, Angaben zur rechtfertigenden Indikation nach § 23 Abs. 1 S. 1, bei einer Untersuchung zusätzlich den erhobenen Befund, die Strahlenexposition des Patienten, soweit sie erfasst worden ist, oder die zu deren Ermittlung erforderlichen Daten und Angaben und bei einer Behandlung zusätzlich den Bestrahlungsplan nach $\S 27$ Abs. 1 S. 1 und das Bestrahlungsprotokoll nach $\S 27$ Abs. 3 enthalten. Minderjährige genießen darüber hinaus einen besonderen Schutz. Ihre Röntgenbilder und Aufzeichnungen von Rönt- genuntersuchungen sind nach $\S 28$ Abs. 3 S. 3 RöV bis zur Vollendung ihres 28. Lebensjahres aufzubewahren.

\section{Folgen einer nichtordnungs- gemäßen Dokumentation}

Verstößt der Behandelnde gegen die ordnungsgemäße Behandlungsdokumentation und verletzt damit eine Pflicht aus dem Behandlungsvertrag, so macht er sich schadensersatzpflichtig. Der Verlust oder die Nichtauffindbarkeit der Patientenakte führt gemäß §630h Abs. 3 BGB zu einer Beweiserleichterung zugunsten des Patienten. Hat in einem zivilrechtlichen Prozess grundsätzlich derjenige, der sich auf einen (Schadensersatz-) Anspruch beruft, mithin der Patient, die Darlegungs- und Beweislast für die anspruchsbegründenden Tatsachen, u. a. auch für den Behandlungs- oder Aufklärungsfehler, so wird nach $\S 630$ h Abs. 3 $B G B$ vermutet, dass eine dokumentationspflichtige medizinisch gebotene wesentliche Maßnahme nicht stattgefunden hat, wenn der Behandelnde die Patientenakte nicht ordnungsgemäß aufbewahrt hat. Der Behandelnde hat sodann den Gegenbeweis gegen die Vermutung zu führen.

\section{Umgang mit Patientenakten bei Praxisaufgabe und -verkauf}

Gibt der behandelnde Arzt seine Praxis aus Altersgründen oder einem anderen Grund auf oder überträgt er sie auf einen Rechtsnachfolger, so enden damit seine Aufbewahrungspflichten nicht.

Auch der Arzt, der seine Praxis ohne Rechtsnachfolger aufgibt, hat seine ärztlichen Aufzeichnungen und Untersuchungsbefunde unter Wahrung der Fristen aufzubewahren oder dafür Sorge zu tragen, dass sie in gehörige Obhut gelangen (vgl. §10 Abs. 4 MBO-Ä). Das schließt auch den Schutz vor unbefugtem Zugriff Dritter mit ein. Der Arzt kann diese Pflicht durch das Anmieten einer Lagerstätte, auf die kein Dritter Zugriff hat, oder die Verwahrung in seinen eigenen Privaträumen entsprechen. Erst nach Ablauf der oben genannten Aufbewahrungsfristen darf der Arzt die Behandlungsdokumentation vernichten. Verstirbt der
Behandelnde vor Ablauf der Aufbewahrungsfrist, so gehen die Pflichten im Wege der Gesamtrechtsnachfolge gemäß § 1922 $\mathrm{BGB}$ auf seine Erben über. Existieren keine Erben oder schlagen diese die Erbschaft aus, so obliegt es gemäß §1936 BGB grundsätzlich dem Staat, die Aufbewahrung der Patientenakten sicherzustellen. Bei radiologischen Behandlungsdokumentationen kann die zuständige Behörde verlangen, dass im Falle der Praxisaufgabe oder sonstiger Einstellung des Betriebes die Aufzeichnungen (und Röntgenbilder) unverzüglich bei einer von ihr bestimmten Stelle zu hinterlegen sind ( $\S 28$ Abs. 3 S. 3 RöV, § 85 Abs. 3 S. 2 StrlSchV).

Folgende Maßnahmen zur Verwahrung der Patientenunterlagen nach Praxisbeendigung sind anerkannt und durch die Bundesärztekammer empfohlen:

a) Aufbewahrung in eigenen Räumen,

b) Aufbewahrung in angemieteten Räumen,

c) Übergabe der Aufzeichnungen an einen Praxisnachfolger,

d) Aushändigung der Aufzeichnungen an den jeweiligen Patienten,

e) Übergabe der Aufzeichnungen an einen anderen niedergelassenen Arzt im Einzugsbereich unter Abschluss eines entsprechenden Verwahrungsvertrages,

f) Übergabe der Unterlagen an ein privates Archivunternehmen, wenn die Mitarbeiter vertraglich zur Verschwiegenheit verpflichtet sind und die Auskunftserteilung aus den Arztaufzeichnungen einem Arzt vorbehalten ist.

Überträgt der Arzt seine Praxis auf einen Rechtsnachfolger, so ist Gegenstand des Praxisverkaufs auch der ideelle Wert einer Praxis (immaterieller Praxiswert oder „Goodwill“). Unter dem ideellen Wert (Goodwill oder immaterieller Praxiswert) versteht man die Chance, eine eingeführte Arztpraxis mit ihrem Patienten- oder Überweiserstamm wirtschaftlich erfolgreich fortzuführen (vgl. Hinweise zur Bewertung von Arztpraxen, DÄBI. 2008, A 2778). Die Patientenakten dürfen dem Praxisnachfolger jedoch nicht ohne Zustimmung des Patienten überlassen werden. Eine uneingeschränkte vertragliche Verpflichtung eines Arztes zur Übertragung des Eigentums an den Patientenakten an den Praxisnach- 
folger ist nichtig, da sie den Patienten in seinem Recht auf informationelle Selbstbestimmung verletzt. Der Bundesgerichtshof $(\mathrm{BGH})$ hat in einer Grundsatzentscheidung (Urteil v. 11.12.1991, Az.: VIII ZR 4/91) entschieden, dass der behandelnde Arzt verpflichtet ist, die Zustimmung des Patienten zu einer Weitergabe seiner Behandlungsdokumentation in eindeutiger und unmissverständlicher Weise einzuholen. Die Annahme eines stillschweigenden oder schlüssig erklärten Einverständnisses oder die mutmaßliche Einwilligung des Patienten ist nicht ausreichend, um die rechtmäßige Übergabe der Patientenakten an den Praxisnachfolger zu gewährleisten. Ein Verstoß kann weitreichende, auch strafrechtliche, Konsequenzen haben. Wer als Arzt unbefugt ein fremdes Geheimnis, das zum persönlichen Lebensbereich eines Patienten gehört, offenbart, das ihm - im Rahmen der Behandlung - anvertraut worden ist, kann mit einer Freiheitsstrafe bis zu einem Jahr oder mit einer Geldstrafe bestraft werden (§ 203 Abs. 1 Nr. 1 StGB).

Empfehlungswert und zweckmäßig ist es daher, bereits in dem Praxiskaufvertrag den rechtmäßigen Umgang mit den Patientenakten zu vereinbaren und die wechselseitigen Rechte und Pflichten von Käufer und Verkäufer zu regeln. Die Übertragung des Eigentums und die sich daraus ergebende uneingeschränkte Verfügungsbefugnis über die Patientenakte sind an die Bedingung zu knüpfen, dass der jeweilige Patient der Weitergabe seiner Behandlungsdokumentation zustimmt. Nach § 4 Abs. 2 BDSG bedarf die Zustimmung des Patienten zur Übergabe seiner Unterlagen an einen Praxisnachfolger grundsätzlich der Schriftform. Zur Sicherstellung kann in dem Praxiskaufvertrag eine Klausel integriert werden, die den Verkäufer der Praxis verpflichtet, seinen Patienten, sobald dieser die Praxis das nächste Mal aufsucht, über den Verkauf der Praxis an den Käufer durch Aushang in den Praxisräumen oder persönliches Anschreiben zu informieren und dessen Zustimmung zur Übergabe seiner Patientendokumentation an den Praxisnachfolger einzuholen. Möglich, aber zeitund kostenintensiv, ist es, den Patienten schriftlich um Erteilung seiner Zustimmung zur Weitergabe seiner Patientenakte zu bitten.

Stimmt der Patient bis zum Übertragungsstichtag der Praxis der Weitergabe seiner Behandlungsdokumentation an den Praxisnachfolger nicht zu, so geht die Patientenakte nicht in das Eigentum des Praxisnachfolgers über. Er darf auf diese keinen Zugriff nehmen oder sie in seine sonstige Patientenkartei einpflegen. Der Käufer kann sich aber vertraglich verpflichten, die Patientenunterlagen - in Papierform oder im EDV-System gespeichert - für den Verkäufer nach den zivilrechtlichen Regelungen (un-) entgeltlich gemäß § 688 ff. BGB in Verwahrung zu nehmen. Die Unterlagen von Patienten, die ihre Zustimmung zur Weitergabe nicht erteilt haben, sind getrennt von den Unterlagen, für die der jeweilige Patient bereits seine Zustimmung zur Einsichtnahme und weitere
Verwendung durch den Praxisnachfolger erteilt hat, in einem separaten Aktenschrank aufzubewahren. Gleichzeitig hat der Käufer sicherzustellen, dass die Behandlungsdokumentation, sofern die Zustimmung nicht erteilt wurde, durch besondere Schutzmaßnahmen organisatorischer und technischer Art vor dem unbefugten Zugriff Dritter bewahrt wird. Diese Pflicht ergibt sich aus § 10 Abs. 4 S. 2 MBO-Ä, der normiert, dass Aufzeichnungen bei einer Praxisübergabe unter Verschluss zu halten sind und nur mit Einwilligung der Patientin oder des Patienten einzusehen oder weiterzugeben sind. Elektronische Patientenakten sind daher durch ein entsprechendes Passwort vor dem unerlaubten Zugriff Dritter zu schützen. Dieses vom BGH entwickelte sog. „Zwei-SchrankModell“ sollte in den Praxiskaufvertrag als vertragliche Verpflichtung des Käufers aufgenommen werden, da den Verkäufer gemäß § 10 Abs. 4 S. 1 MBO-Ä die berufsrechtliche Pflicht trifft, die Patientenakten in gehörige Obhut zu geben. Diese Pflicht gewährleistet er, in dem er die Patientenakten dem Praxisnachfolger zur Verwahrung überlässt und ihn anweist, diese entsprechend der gesetzlichen Anforderungen aufzuheben.

Die Zustimmungserklärung des Patienten ist in der Patientenakte zu dokumentieren. Erst nach Erteilung der Zustimmung darf der Praxisnachfolger die Patientenakte aus dem separaten Aktenschrank entnehmen, Einsicht nehmen und in seine sonstige Praxiskartei aufnehmen.

\title{
NEU: IHR PERSONALISIERTES PATIENTEN-MAGAZIN FÜR IHRE KLINIK ODER PRAXIS
}

\author{
Ihr Corporate Design, Ihre \\ Bilder \& Ihre Inhalte - von \\ uns druckfertig aufbereitet
}

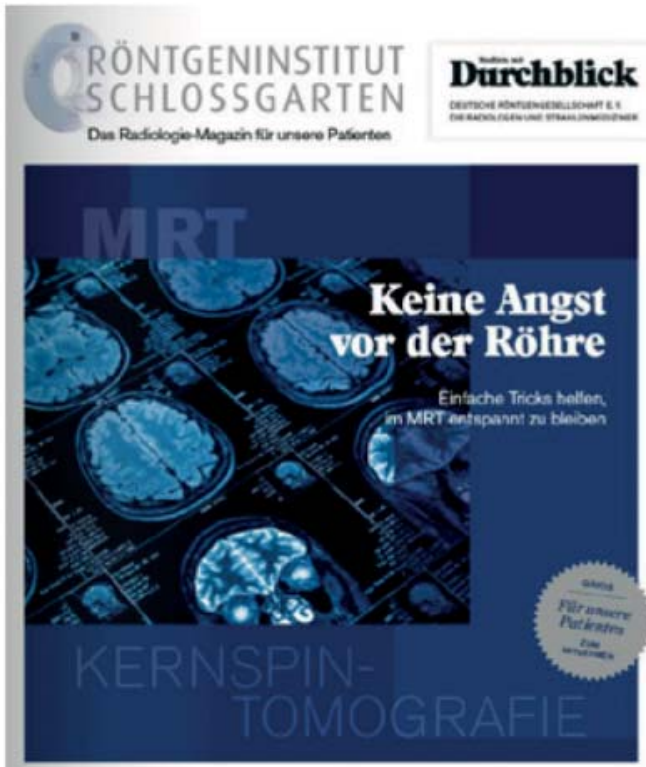


der Gesellschaft gemäß §630g Abs. 2 BGB elektronische Abschriften oder wahlweise Fotokopien von seiner Patientenakte verlangen und diese dann dem Ausgeschiedenen zur weiteren Verwendung zur Verfügung stellen. Dabei ist der Patient jedoch verpflichtet, der Gesellschaft die durch die Erstellung der Abschriften oder Kopien anfallenden Kosten zu erstatten. Eine Pflicht der Gesellschaft zur Herausgabe der Originalunterlagen besteht nicht. Der ausgeschiedene Radiologe, der den Patienten weiterbehandelt, kann von der Gesellschaft verlangen, ihm die Aufzeichnungen und Röntgenbilder vorübergehend zu überlassen (§ 28 Abs. 8 S. 1 RöV). Die Vorschrift enthält eine Überlassensregelung insbesondere mit dem Ziel, einem weiter- oder mitbehandelnden Arzt die Voruntersuchungen zugänglich zu machen, um unnötiges Mehrfachröntgen zu vermeiden. Die Verwendung des Begriffes „vorübergehend“ in Satz 1 und 2 stellt klar, dass die Unterlagen in jedem Fall an den nach Abs. 3 Aufbewahrungspflichtigen zurückzugeben sind. Dieser soll sich nicht durch Weitergabe der Unterlagen seiner Aufbewahrungspflicht entledigen können (vgl. Verordnung zur Änderung der Röntgenverordnung und anderer atomrechtlicher Verordnungen, BRDrucks. 230/02v. 13.03.02, B Zu Nr. 45 (§ 28) S. 96).

Wird die Gesellschaft aufgelöst und abgewickelt und beabsichtigt der behandelnde Arzt seine Tätigkeit und die Behandlung des jeweiligen Patienten anderweitig fortzusetzen, so hat er nicht das Recht, die Behandlungsdokumentation des Patienten an sich zu nehmen. Da es der Gesellschaft als Behandelnde im Sinne des Gesetzes obliegt, die Behandlungsdokumentation aufzubewahren, bedarf es bei einer Übertragung der Patientenakte auf den behandelnden Arzt der Zustimmung des Patienten. Dieser ist über die Auflösung der Gesellschaft zu informieren und hat die Gesellschaft in Kenntnis zu setzen, welcher Arzt aus der Gesellschaft seine Behandlung zukünftig fortsetzen und wer seine Patientenunterlagen und die sich daraus ergebenden Rechte und Pflichten übernehmen soll. Sofern vor der endgültigen Auseinandersetzung der Gesellschaft keine verbindliche Mitteilung des Patienten vorliegt, wie mit seiner Patientenakte zu verfahren ist, sollte zwischen den Gesellschaftern ein einheitli- cher Aufbewahrungsort für alle Patientenakten gewählt werden, um zu garantieren, dass der Patient, falls gewünscht, im Rahmen der Aufbewahrungsfristen Zugriff auf seine Behandlungsdokumentation erhalten kann.

\section{Fazit}

Der behandelnde Arzt hat bei einer Praxisaufgabe oder einem Praxisverkauf umfassende rechtliche Vorgaben zu beachten, wie mit seiner Behandlungsdokumentation umzugehen ist. Eine auch unwissentliche Nichtbeachtung der Aufbewahrungspflichten kann neben zivilrechtlichen Konsequen- zen auch die Gefahr bergen, sich einer Strafverfolgung auszusetzen.

Prof. Dr. Peter Wigge

Rechtsanwalt

Fachanwalt für Medizinrecht

Dina Gebhardt

Rechtsanwältin

Rechtsanwälte Wigge

Scharnhorststraße 40

48151 Münster

Tel.; ++49/251/535 950

Fax: ++ 49/251/5359599

kanzlei@ra-wigge.de

\section{Ihre Vorteile einer DRG-Mitgliedschaft}

Inhaltlich MITGESTALTEN

Aktive Beteiligung an der inhaltlichen Arbeit und Entwicklung des Fachs in Arbeitsgemeinschaften, Foren und Programmen.

\section{Umfassend INFORMIEREN}

Immer up to date durch Publikationen, Periodika und News-Services.

Persönlich VERNETZEN

Fachlicher Austausch zu aktuellen Themen im Mitgliederbereich von drg,de, auf Veranstaltungen und Arbeitstreffen.

Bedarfsbezogen NUTZEN

Angebote und Leistungen in Anspruch nehmen wie z. B. die Zertifizierungsprogramme der DRG und ihrer Arbeitsgemeinschaften.

Praktisch ORGANISIEREN

Zusatzfunktionen und Services nutzen wie z. B. den individualisierten RöKo-Kongressplaner.

\section{Finanziell PROFITIEREN}

Weniger bezahlen bei einer Teilnahme am Deutschen Röntgenkongress und weiteren Veranstaltungen der DRG, für Mitgliedschaften in internationalen Vereinigungen, den Bezug von Zeitschriften und für Produkte unserer Kooperationspartner.

( $($ W Weiterführende Informationen finden Sie hier: www.drg.de > Mitglieder 